\title{
Silencing of PFKFB3 protects podocytes against high glucose-induced injury by inducing autophagy
}

\author{
ZHENGMING ZHU ${ }^{1}$, QINGSHENG LIU ${ }^{2}$, JIANSHI SUN ${ }^{3}$, ZIYANG BAO ${ }^{1}$ and WEIWEI WANG ${ }^{1}$ \\ Departments of ${ }^{1}$ Nephrology and ${ }^{2}$ Geriatrics, Hangzhou Hospital of Traditional Chinese Medicine, \\ Hangzhou, Zhejiang 310007; ${ }^{3}$ Department of Nephrology, Guang'anmen Hospital, \\ China Academy of Chinese Medical Sciences, Beijing 100053, P.R. China
}

Received September 27, 2020; Accepted May 19, 2021

DOI: $10.3892 / \mathrm{mmr} .2021 .12405$

\begin{abstract}
Diabetic nephropathy (DN) is a diabetic complication that threatens the health of patients with diabetes. In addition, podocyte injury can lead to the occurrence of DN. The protein 6-phosphofructo-2-kinase/fructose-2,6-biphosphatase 3 (PFKFB3) may be associated with diabetes; however, the effects of PFKFB3 knockdown by small interfering (si)RNA on the growth of podocytes remains unknown. To investigate the mechanism by which PFKFB3 mediates podocyte injury, MPC5 mouse podocyte cells were treated with high-glucose (HG), and cell viability and apoptosis were examined by Cell Counting Kit- 8 assay and flow cytometry, respectively. In addition, the expression of autophagy-related proteins were measured using western blot analysis and immunofluorescence staining. Cell migration was investigated using a Transwell assay and phalloidin staining was performed to observe the cytoskeleton. The results revealed that silencing of PFKFB3 significantly promoted MPC5 cell viability and inhibited apoptosis. In addition, the migration of the MPC5 cells was notably downregulated by siPFKFB3. Moreover, PFKFB3 silencing notably reversed the $\mathrm{HG}$-induced decrease in oxygen consumption rate, and the $\mathrm{HG}$-induced increase in extracellular acidification rate was rescued by PFKFB3 siRNA. Furthermore, silencing of PFKFB3 induced autophagy in HG-treated podocytes through inactivating phosphorylated (p-)mTOR, p-AMPK $\alpha$, LC3 and sirtuin 1, and activating p62. In conclusion, silencing of PFKFB3 may protect podocytes from HG-induced injury by inducing autophagy. Therefore, PFKFB3 may serve as a potential target for treatment of DN.
\end{abstract}

Correspondence to: Dr Zhengming Zhu, Department of Nephrology, Hangzhou Hospital of Traditional Chinese Medicine, 453 Tiyuchang Road, Hangzhou, Zhejiang 310007, P.R. China E-mail: zzmdoctor@163.com

Key words: diabetic nephropathy, 6-phosphofructo-2kinase/fructose-2,6-biphosphatase 3, autophagy, high glucose, podocyte

\section{Introduction}

Diabetic nephropathy (DN) is a common diabetic complication, the incidence of which was $20-40 \%$ among patients with diabetes in 2019 worldwide, which develops from permanent uncontrolled diabetes. Furthermore, it typically causes end-stage renal disease (1). DN is characterized, clinically, by hypertension, progressive proteinuria and renal disease resulting in multiple diseases, including uremic symptoms (2). The main characteristics of DN are increased thickness of the basement membrane and mesangial expansion in the glomerulus resulting from the accumulation of the extracellular matrix, followed by hypercellularity, which ultimately leads to glomerular sclerosis and renal fibrosis (3-5). As high-glucose (HG)-induced injury of podocytes has been associated with DN progression (6), it is important to identify novel strategies for protecting podocytes from injury against $\mathrm{HG}$.

Increased glycolysis in podocytes has been regarded as a primary cause of DN (7). One of the key mediators and the first committed factor of glycolytic flux is 6-phosphofructo-2-kinase/fructose-2,6-biphosphatase 3 (PFKFB3) (8). PFKFB3 serves a role in the synthesis and degradation of fructose-2,6-bisphosphate, which has been associated with diabetes (9), cell cycle mediation (10) and drug resistance (11). However, the biological function of PFKFB3 in podocyte injury remains unclear.

Autophagy is a cellular process that is characterized by the formation of an isolation membrane called a phagophore (12). Numerous studies have indicated that autophagy serves an important role in cell growth and differentiation $(13,14)$. Furthermore, autophagy is associated with the progression of various diseases, including Alzheimer's disease (15), cancer (16) and microorganism infection (17). In addition, it has been previously reported that induction of autophagy protects podocytes from injury (18). Therefore, the aim of the present study was to investigate the association between PFKFB3 and autophagy. Enolase-1, glyoxalase and aldose reductase (AR) are considered three important markers in $\mathrm{DN}$, and the regulation of these three factors may modulate the progression of DN $(19,20)$. Therefore, AR was used as a positive control to study the effect of PFKFB3 siRNA on enolase-1 and glyoxalase. The effects of PFKFB3 small interfering (si)RNA on the growth of HG-induced podocytes 
in vitro was also determined to identify novel targets for the treatment of DN.

\section{Materials and methods}

Cell culture and treatment. MPC5 mouse podocyte cells were purchased from the Tongpai Biotechnology Co. Ltd. RPMI-1640 (Gibco; Thermo Fisher Scientific, Inc.) medium containing 10\% FBS (Gibco; Thermo Fisher Scientific, Inc.), $1 \%$ penicillin and streptomycin and $10 \mathrm{IU} / \mathrm{ml}$ recombinant murine IFN- $\gamma$ (Invitrogen; Thermo Fisher Scientific, Inc.) was utilized to culture MPC5 cells at $37^{\circ} \mathrm{C}$ and $5 \% \mathrm{CO}_{2}$. Podocytes were maintained at $37^{\circ} \mathrm{C}$ in the absence of IFN- $\gamma$ for $10-14$ days to induce differentiation, and then cultured for $12 \mathrm{~h}$ at $37^{\circ} \mathrm{C}$ in FBS-free RPMI-1640 medium containing 5.6 mM D-glucose (Sigma-Aldrich; Merck KGaA) before subsequent experiments and cell transfection. In addition, MPC5 cells were treated with $25 \mathrm{mM}$ glucose (HG; Sigma-Aldrich; Merck KGaA) or $10 \mathrm{mM}$ glucose [normal glucose (NG); Sigma-Aldrich; Merck KGaA] at $37^{\circ} \mathrm{C}$ for $48 \mathrm{~h}$. In order to maintain the osmotic pressure, MPC5 cells were treated with $19.5 \mathrm{mM}$ mannitol (Sigma-Aldrich; Merck KGaA) at $37^{\circ} \mathrm{C}$ for $48 \mathrm{~h}$.

Human primary podocytes (cat. no. BNCC340460; BeNa Culture Collection; Beijing Beina Chunglian Institute of Biotechnology) were cultured at $33^{\circ} \mathrm{C}$ in RPMI-1640 medium with 10\% FBS (both Gibco; Thermo Fisher Scientific, Inc.), $100 \mu \mathrm{g} / \mathrm{ml}$ streptomycin, $100 \mathrm{U} / \mathrm{ml}$ penicillin $\mathrm{G}$ and $1 \mathrm{X}$ insulin-transferrin-selenium (ITS; Invitrogen) for $48 \mathrm{~h}$. To induce differentiation, cells were cultured at $37^{\circ} \mathrm{C}$ for $10-14$ days with ITS-free medium. The differentiated cells were stimulated for $48 \mathrm{~h}$ at $37^{\circ} \mathrm{C}$ with $\mathrm{NG}(10 \mathrm{mM})$ or $\mathrm{HG}(25 \mathrm{mM})$.

Reagents. Chloroquine (CQ) and rapamycin are known autophagy inhibitors; thus, they can be used in rescue experiments to further confirm the role of PFKFB3 in autophagy. $\mathrm{CQ}$ and rapamycin were obtained from Sigma-Aldrich (Merck KGaA). To investigate the role of PFKFB3 in cell autophagy, MPC5 cells were treated with CQ $(5 \mathrm{mM})$ or rapamycin $(10 \mu \mathrm{M})$ for $48 \mathrm{~h}$ at $37^{\circ} \mathrm{C}$.

Cell transfection. Cell transfection was performed following HG treatment. MPC5 cells or primary podocytes were seeded at $3 \times 10^{5}$ cells/well in a 6 -well plate and cultured to $70 \%$ confluence. The cells were subsequently transfected with AR siRNA (10 nM), PFKFB3 siRNA1 (10 nM), siRNA2 (10 nM) or siRNA3 $(10 \mathrm{nM})$, or negative control (NC, $10 \mathrm{nM})$ siRNA using Lipofectamine ${ }^{\circledR} 2000$ reagent (Thermo Fisher Scientific, Inc.) for 24,48 or $72 \mathrm{~h}$ at $37^{\circ} \mathrm{C}$. The siRNAs targeting AR, PFKFB3 and NC siRNA were designed and synthesized by Shanghai GenePharma Co., Ltd.; the sequences were as follows: AR siRNA, 5'-GCAATCTGAGTCTCCAACT-3'; PFKFB3 siRNA1, 5'-GCAAUUGAAAGUUUGGUAA-3'; PFKFB3 siRNA2, 5'-CCCACA ACCUUUAGACUGA-3'; PFKFB3 SiRNA3, 5'-GAAUCCAUAUCACUACGAA-3'; and NC siRNA, 5'-GUACAGAGUUACUACUUAG-3'. After $48 \mathrm{~h}$ of transfection, the transfected cells were used in subsequent analysis.

Cell Counting Kit (CCK)-8 assay. MPC5 cells or primary podocytes were seeded in 96 -well plates $\left(5 \times 10^{3}\right.$ cells/well) overnight at $37^{\circ} \mathrm{C}$. Then, the cells were treated with $\mathrm{NC}$ siRNA + NG, AR siRNA + NG, PFKFB3 siRNA + NG, NC siRNA + HG, AR siRNA + HG or PFKFB3 siRNA for $48 \mathrm{~h}$, as aforementioned. A total of $10 \mu \mathrm{lCCK}-8$ reagent (Beyotime Institute of Biotechnology) was added to each well and incubated for $2 \mathrm{~h}$ at $37^{\circ} \mathrm{C}$. Finally, the absorbance of MPC5 cells was measured at $450 \mathrm{~nm}$ using a microplate reader (Thermo Fisher Scientific, Inc.).

Glucose uptake detection. The supernatant of MPC5 cells was collected by centrifugation at $100 \mathrm{xg}$ at $4^{\circ} \mathrm{C}$ for $10 \mathrm{~min}$, then the concentration of glucose and diacylglycerol (DAG) was detected as previously described (21). The absorbance of MPC5 cell supernatants was measured at $505 \mathrm{~nm}$ using a microplate reader (Thermo Fisher Scientific, Inc.). The concentration was calculated as previously described (21).

Oxygen consumption rate (OCR) and extracellular acidification rate (ECAR) measurement. OCR measurements were performed on a Seahorse XFp analyzer using the Seahorse XF Cell Mito Stress Test kit (both Agilent Technologies, Inc.) according to the manufacturer's instructions. Briefly, podocytes were seeded on XFe96 plates at $5 \times 10^{4}$ cells/well. OCR was measured following exposure to mitochondrial modulators as follows: Oligomycin $(1 \mu \mathrm{M})$, which inhibits ATP synthase and decreases OCR; carbonyl cyanide 4-trifluoromethoxyphenylhydrazone $(1.5 \mu \mathrm{M})$, which uncouples oxygen consumption from ATP production and raises OCR to a maximal value; and a combination of rotenone and antimycin A (both $0.5 \mu \mathrm{M}$ ), which decrease OCR to a minimal value. After the OCR readings were completed, cells were fixed in $4 \%$ paraformaldehyde at room temperature for 10 min and stained with a $0.05 \%$ crystal violet solution at room temperature for $30 \mathrm{~min}$. Next, podocytes were washed and lysed in $1 \%$ SDS. Absorbance of each well (which reflects cell number) was read at $595 \mathrm{~nm}$ using an Infinite 200 Pro plate reader (Tecan Group Ltd.). OCR values were normalized to cell number.

For ECAR measurement, podocytes $\left(3 \times 10^{5}\right.$ per well) were cultured on 8-well culture microplates (Agilent Technologies, Inc.) coated with Rat Tail Collagen-I Solution (Sigma-Aldrich; Merck KGaA) overnight. Next, growth medium was replaced with assay medium (minimal DMEM supplemented with 2 mM L-glutamine; Gibco; Thermo Fisher Scientific, Inc.) and cells were allowed to stabilize at $37^{\circ} \mathrm{C}$ for $1 \mathrm{~h}$. ECAR values were determined using the Seahorse XFp Analyzer before (baseline state) and after cells were treated with $10 \mathrm{mM}$ D-glucose, $1 \mu \mathrm{M}$ oligomycin or $50 \mathrm{mM} 2$-deoxy-D-glucose. Glycolytic flux parameters were determined from the slopes of the ECAR values in real-time analysis. Baseline and post-exposure rates were measured three times every $3 \mathrm{~min}$ (total time, $\sim 80 \mathrm{~min}$ ). The results were normalized to the protein concentrations determined for each cell culture plate well using the Bradford method.

Cell apoptosis analysis. Cell apoptosis was detected using Annexin V-FITC/PI apoptosis detection kit (cat. no. 556570; BD Biosciences). MPC-5 cells or primary podocytes were seeded in a 6 -well plate $\left(1 \times 10^{6} /\right.$ well $)$ at $37^{\circ} \mathrm{C}$ overnight. Cells were trypsinized, then washed, fixed with $70 \%$ ethanol at $4^{\circ} \mathrm{C}$ 
for $1 \mathrm{~h}$ and re-suspended in Annexin V Binding Buffer. Subsequently, the cells were stained with $5 \mu \mathrm{l}$ FITC and PI (BD Biosciences) in the dark at $4^{\circ} \mathrm{C}$ for $15 \mathrm{~min}$. The rate of early + late-stage cell apoptosis was calculated using a flow cytometer (BD Biosciences), and the results were analyzed by flow cytometry (FACSCalibur; BD Biosciences) with FlowJo (v10.6.2; FlowJo LLC).

Western blot analysis. Total protein was extracted from cell lysate using RIPA buffer (Beyotime Institute of Biotechnology) and quantified using a BCA protein kit (Thermo Fisher Scientific, Inc.). Proteins (40 $\mu \mathrm{g}$ per lane) were separated using SDS-PAGE (10\%) and transferred onto PVDF membranes. Subsequently, the membranes were blocked with $3 \%$ skimmed milk at room temperature for $1 \mathrm{~h}$, incubated with primary antibodies at $4^{\circ} \mathrm{C}$ overnight, then incubated with HRP-conjugated secondary anti-rabbit antibody (1:5,000; cat. no. ab7090; Abcam) at room temperature for $1 \mathrm{~h}$. The membranes were scanned using an Odyssey Imaging System and data was analyzed using ImageJ software (version 1.8.0; National Institutes of Health). The primary antibodies were as follows: Anti-synaptopodin (cat. no. DF12173; Affinity Biosciences), anti-enolase-1 (cat. no. ab227978; Abcam), anti-PFKFB3 (cat. no. DF3307; Affinity Biosciences), anti-glyoxalase 1 (cat. no. DF6700; Affinity Biosciences), anti-AR (cat. no. DF6554; Affinity Biosciences), anti-Wilms tumor 1 (WT-1;cat. no. 12609-1-AP; ProteinTech Group, Inc.), anti-E-cadherin (cat. no. ab40772; Abcam), anti- $\alpha$-smooth muscle actin (SMA; cat. no. ab7817; Abcam), anti-p62 (cat. no. ab109012; Abcam), anti-light chain (LC)3 (cat. no. ab192890; Abcam), anti-phosphorylated (p)-mTOR (cat. no. ab109268; Abcam), anti-mTOR (cat. no. ab32028; Abcam), anti-sirtuin (SIRT)1 (cat. no. ab32441; Abcam), anti-AMPK $\alpha$ (cat. no. ab32047; Abcam), anti-p-AMPK $\alpha$ (cat. no. ab133448; Abcam) and anti-GAPDH (cat. no. ab8245; Abcam) (all 1:1,000). GAPDH was used as an internal control.

Transwell assay. For the migration assay, transfected cells ( $3 \times 10^{4}$ cells/well) in serum-free DMEM $(100 \mu \mathrm{l})$ were seeded into the upper chamber of a Transwell plate (24-wells; pore size, $8 \mu \mathrm{m}$; Corning, Inc.). A total of $600 \mu \mathrm{l}$ DMEM containing $10 \%$ FBS was added into the lower chamber of each well. Following incubation for $24 \mathrm{~h}$ at room temperature, the non-migrated cells on the upper surface were removed, and the migrated cells on the lower surface were fixed with $4 \%$ paraformaldehyde at $4{ }^{\circ} \mathrm{C}$ for $15 \mathrm{~min}$, followed by staining with $0.5 \%$ crystal violet at room temperature for $30 \mathrm{~min}$. The number of migrated cells was calculated using a light microscope, x400 magnification; three random fields of view were selected.

Immunofluorescence staining. Following treatment for $72 \mathrm{~h}$, as aforementioned, MPC-5 cells were blocked with $4 \%$ paraformaldehyde at room temperature for $15 \mathrm{~min}$, then incubated with anti-phalloidin (cat. no. ab176753) or anti-LC3 (cat. no. ab192890) antibody (both 1:1,000; both Abcam) overnight at $4^{\circ} \mathrm{C}$. Subsequently, the cells were incubated with the Goat Anti-Rabbit IgG H\&L antibody (1:5,000; cat. no. ab150077; Abcam) at room temperature for $1 \mathrm{~h}$. DAPI (Beyotime Institute of Biotechnology) was used to stain the nuclei for $5 \mathrm{~min}$. Finally, the cells were observed under a fluorescence microscope (magnification, x200). The fluorescence intensity was measured using ImageJ software (version 1.8.2; National Institutes of Health); a total of three random fields of view were selected.

Statistical analysis. All data are presented as the mean \pm standard error of the mean. The CCK-8 assay was repeated five times; immunofluorescence staining, RT-qPCR, Transwell and flow cytometry assays were repeated three times. Comparisons between two groups were analyzed by unpaired Student's t-test. One-way ANOVA followed by Tukey's post hoc test was used for multiple group comparisons. The normality of data was assessed using Shapiro-Wilk test. Data were analyzed using GraphPad Prism (version 7; GraphPad Software, Inc.). $\mathrm{P}<0.05$ was considered to indicate a statistically significant difference.

\section{Results}

$A R$ and PFKFB3 siRNAs significantly decreased the level of $A R$ and PFKFB3 in MPC5 cells, respectively. To establish an in vitro model of DN, MPC5 cells were treated with HG as previously described $(22,23)$. As AR inhibitors have been shown to inhibit the progression of DN $(24,25)$, AR siRNA was used in preliminary experiments (cell viability, migration and synaptopodin expression). HG treatment significantly increased the protein expression levels of enolase-1, PFKFB3 and AR in MPC5 cells at 48 and 72 h (Fig. 1A). By contrast, the protein expression levels of glyoxalase 1 were significantly downregulated in the presence of $\mathrm{HG}$. As the aforementioned four proteins are key markers of DN (26-29), the results suggested that the in vitro model of DN was successfully established. Next, western blot analysis was performed to detect the transfection efficiency of siRNAs. Knockdown of AR significantly decreased the protein expression level of AR in MPC5 cells (Fig. 1B). Similarly, the expression level of PFKFB3 protein in MPC5 cells was significantly inhibited by silencing of PFKFB3 (Fig. 1B); PFKFB3 siRNA3 showed the most significant transfection efficiency and was selected to be used in the subsequent experiments. mRNA expression of PFKFB3 in primary podocytes was significantly decreased by PFKFB3 siRNA3 (Fig. S1A and B). Taken together, these results indicated that $A R$ and PFKFB3 siRNAs significantly decreased the expression levels of AR and PFKFB3 in MPC5 cells, respectively.

$H G$-induced inhibition of cell viability is reversed by PFKFB3 siRNA. To investigate the effect of PFKFB3 or AR on the viability of MPC5 cells, CCK-8 assay was used. Viability of MPC5 cells in the presence of NG was slightly affected by AR siRNA but was significantly enhanced following PFKFB3 knockdown (Figs. 2A and S2A). In addition, HG significantly inhibited the viability of MPC5 cells. However, PFKFB3 siRNA significantly rescued the inhibitory effect of $\mathrm{HG}$ on cell viability. Mannitol did not affect the viability of HG-treated MPC5 cells. Western blot analysis was used to measure the expression levels of synaptopodin. The protein expression levels of synaptopodin in MPC5 cells in the presence of NG were significantly increased by PFKFB3 silencing (Fig. 2B). HG significantly decreased the protein expression 
A
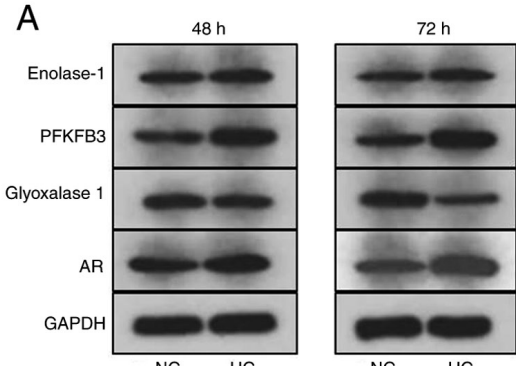

B
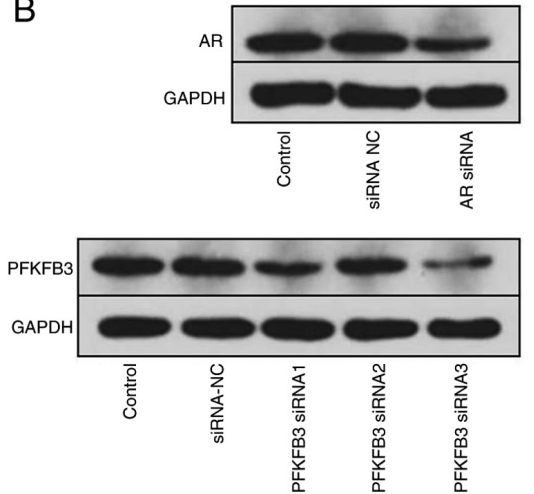

Enolase-1

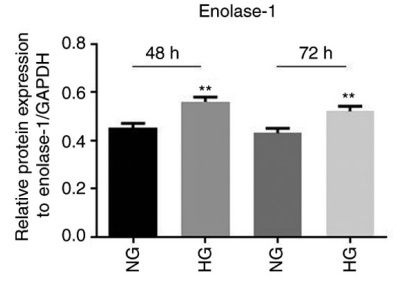

Glyoxalase 1

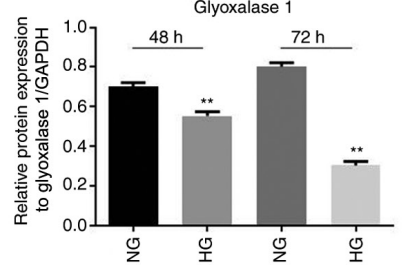

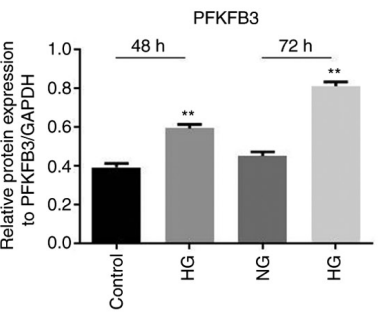

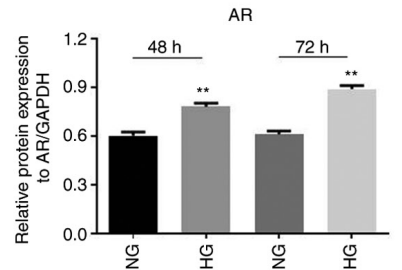

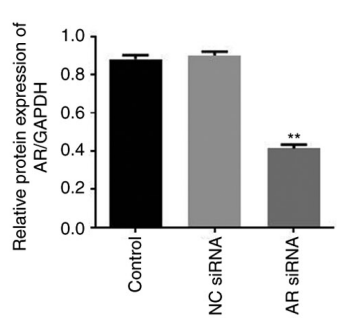

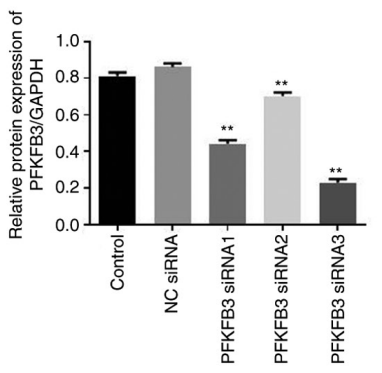

Figure 1. AR and PFKFB3 siRNAs are successfully transfected into MPC5 cells. MPC5 cells were treated with HG for 48 or $72 \mathrm{~h}$. (A) Protein expression levels of enolase-1, PFKFB3, glyoxalase 1 and AR in MPC5 cells were detected by western blot analysis and normalized to GAPDH. (B) MPC5 cells were transfected with siRNA NC, siRNA AR or PFKFB3 siRNA1, -2 or -3 for $24 \mathrm{~h}$. Then, the protein expression levels of PFKFB3 and AR were measured by western blot analysis and normalized to GAPDH. " $\mathrm{P}<0.01$ vs. Control (NG). AR, aldose reductase; HG, high glucose; NC, negative control; PFKFB3, 6-phosphofructo-2-kinase/fructose-2,6-biphosphatase 3; siRNA, small interfering RNA.

A

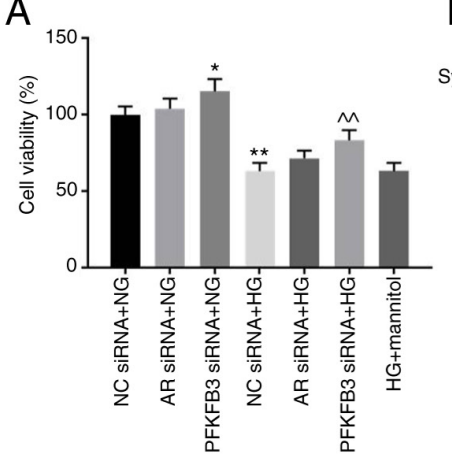

B

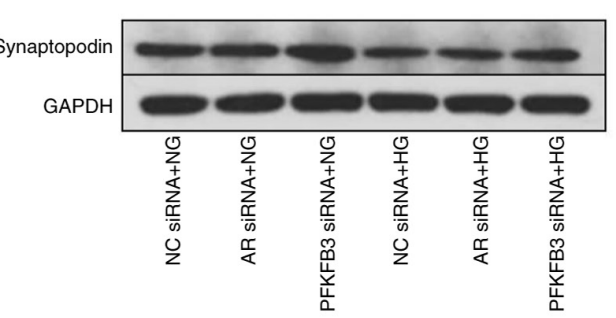

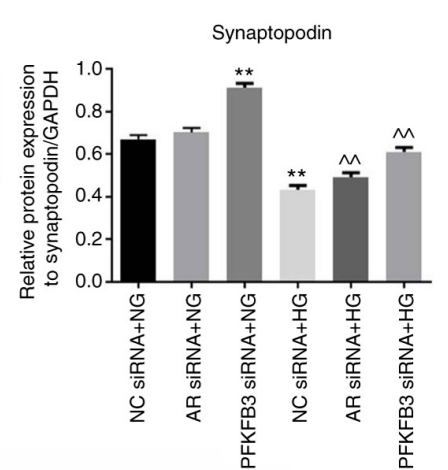

C

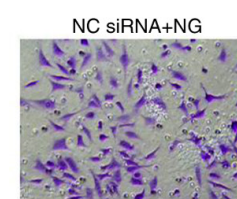

NC siRNA+HG

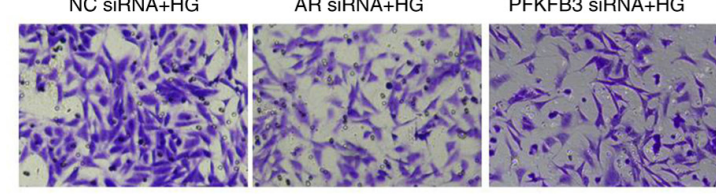

AR siRNA+NG
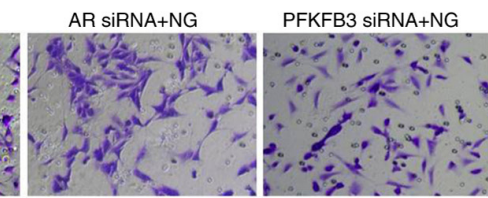

$\therefore \div \frac{2}{2} \times 1$ ?

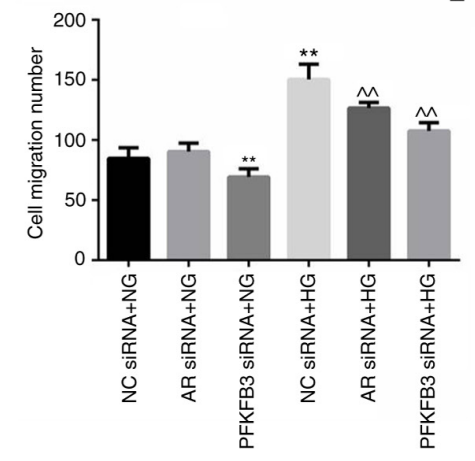

Figure 2. HG-induced cell viability inhibition and cell migration is reversed by PFKFB3 siRNA. MPC5 cells were treated with NC siRNA + NG, AR siRNA + NG, PFKFB3 siRNA + NG, NC siRNA + HG, AR siRNA + HG, PFKFB3 siRNA + HG or HG + mannitol for 48 h. (A) Cell viability was measured by Cell Counting Kit-8 assay. (B) Protein expression levels of synaptopodin in MPC5 cells were detected by western blot analysis and normalized to GAPDH. (C) Cell migration was examined by Transwell assay. ${ }^{*} \mathrm{P}<0.05,{ }^{* *} \mathrm{P}<0.01$ vs. NC siRNA $+\mathrm{NG} ;{ }^{\wedge} \mathrm{P}<0.01 \mathrm{vs}$. NC siRNA $+\mathrm{HG}$. AR, aldose reductase; HG, high-glucose; NC, negative control; NG, normal glucose; PFKFB3, 6-phosphofructo-2-kinase/fructose-2,6-biphosphatase 3; siRNA, small interfering RNA. 

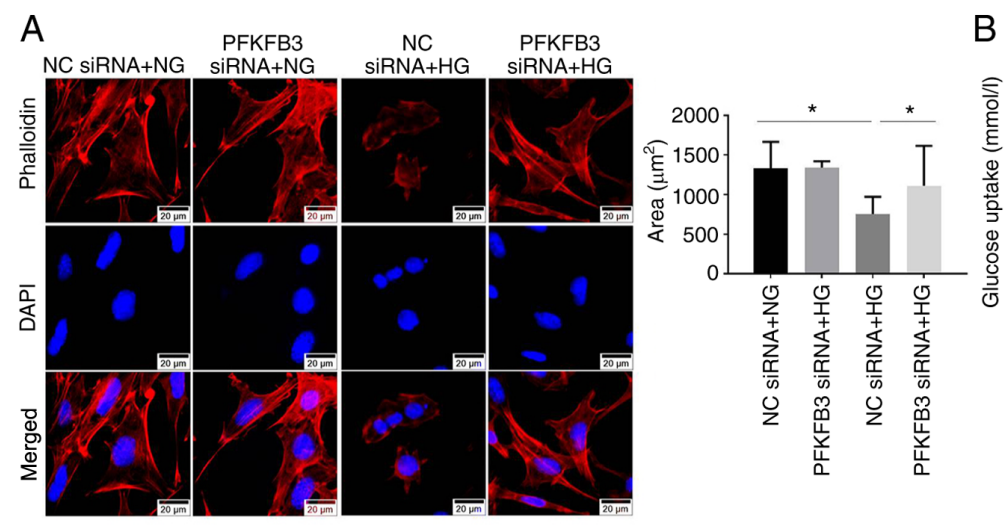

B
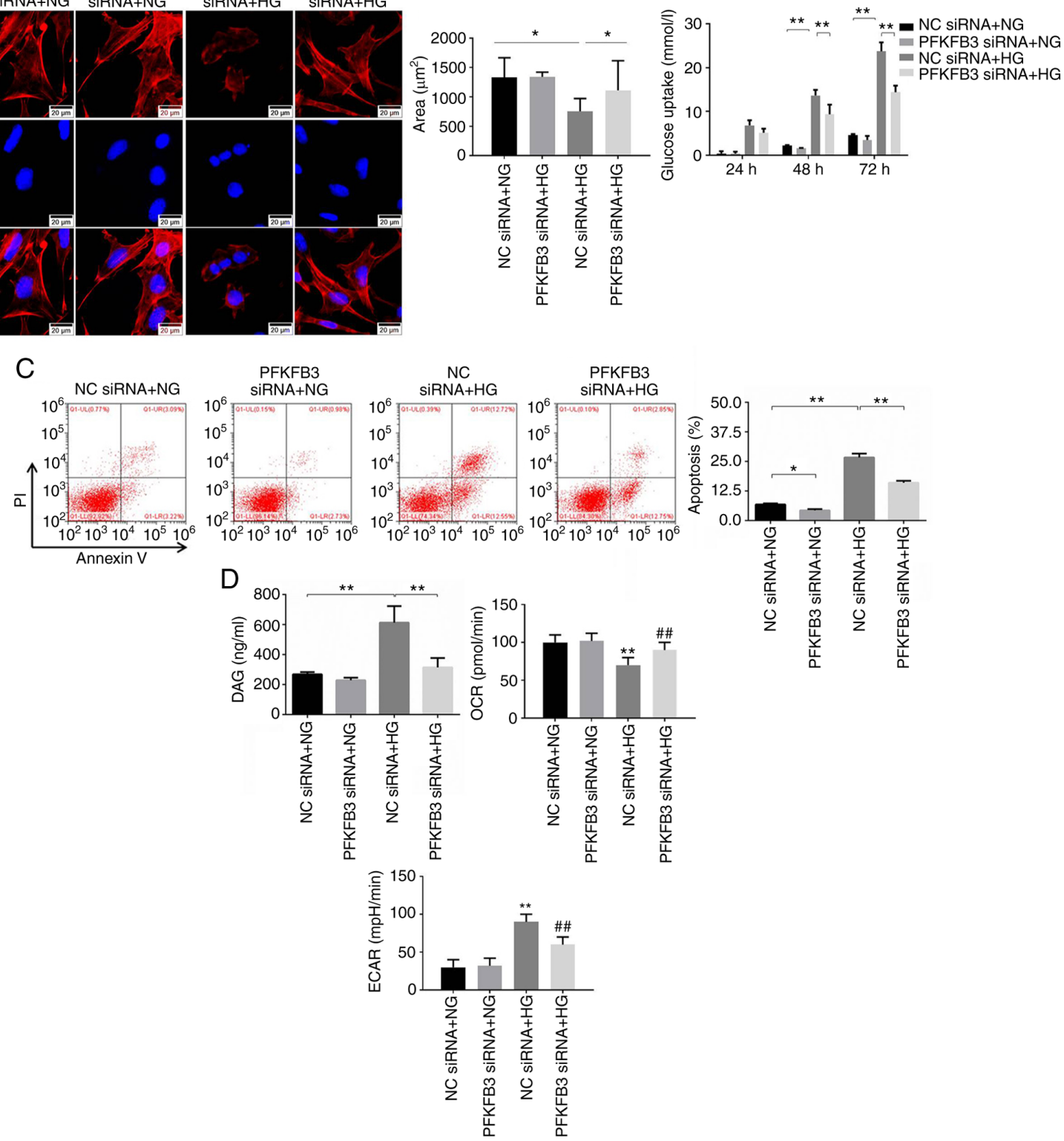

Figure 3. Silencing of PFKFB3 reverses HG-induced podocyte injury. (A) Phalloidin immunofluorescence staining was performed to detect the cytoskeleton, and the area of MPC5 cells was calculated. (B) Glucose uptake in MPC5 cells was tested using a microplate reader. (C) Cell apoptosis was detected by flow cytometry. (D) Levels of DAG in MPC5 cells were detected using a microplate reader. OCR or ECAR in MPC5 cells was investigated by Seahorse analyzer. ${ }^{*} \mathrm{P}<0.05,{ }^{* *} \mathrm{P}<0.01$ vs. NC siRNA + NG or as indicated; ${ }^{\# /} \mathrm{P}<0.01$ vs. NC siRNA + HG. DAG, diacylglycerol; ECAR, extracellular acidification rate; HG, high-glucose; NC, negative control; NG, normal glucose; OCR, oxygen consumption rate; PFKFB3, 6-phosphofructo-2-kinase/fructose-2,6-biphosphatase 3; siRNA, small interfering RNA.

levels, and PFKFB3/AR knockdown partially reversed this inhibitory effect of HG on synaptopodin protein expression levels (Fig. 2B). Transwell assay was performed to assess cell migration; migration of the MPC5 cells was significantly upregulated in the presence of HG compared with NG, which was partially reversed in the presence of AR or PFKFB3 siRNAs (Fig. 2C). Taken together, these results indicated that HG-induced cell viability inhibition, migration and decrease of synaptopodin expression were reversed by PFKFB3 siRNA.

Silencing of PFKFB3 reverses $H G$-induced podocyte injury. Phalloidin staining was performed to examine the cytoskeleton. The results revealed that HG treatment significantly changed the shape and decreased the area of MPC5 cells, whereas knockdown of PFKFB3 significantly rescued the injury caused by HG (Fig. 3A). In addition, glucose uptake by the MPC5 cells was significantly increased after 48 or $72 \mathrm{~h}$ of HG treatment, which was partially inhibited by PFKFB3 siRNA (Fig. 3B). Similarly, PFKFB3 siRNA significantly reversed the HG-induced apoptosis of MPC-5 cells and primary podocytes (Figs. 3C and S2B). Furthermore, DAG concentration and ECAR in MPC5 cells were significantly upregulated by HG; this was partially reversed in the presence of PFKFB3 siRNA (Fig. 3D). By contrast, the HG-induced decrease of OCR in podocytes was significantly reversed in the presence of PFKFB3 siRNA (Fig. 3D). Taken together, these results indicated that silencing of PFKFB3 reversed HG-induced podocyte injury. 
A

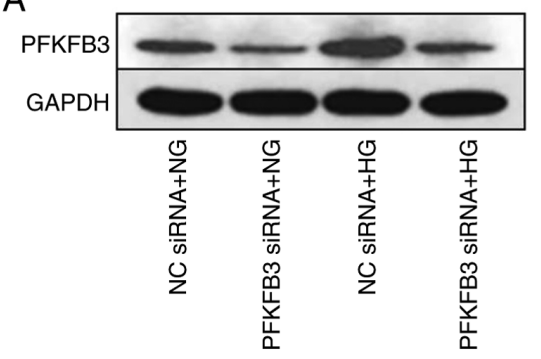

B

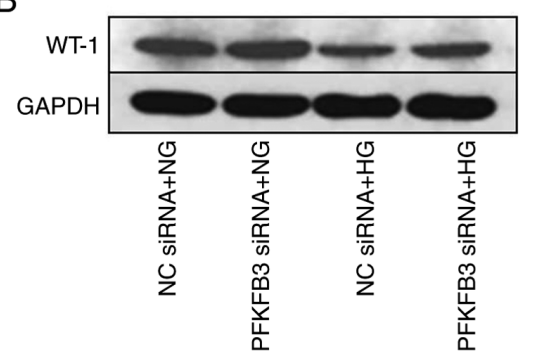

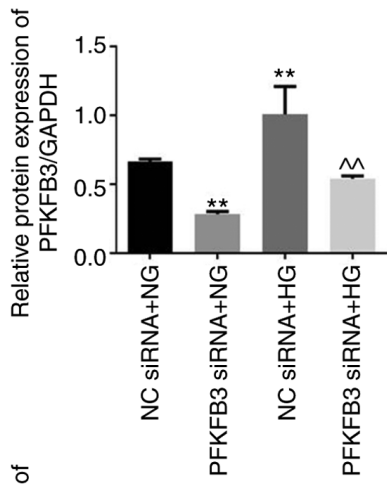

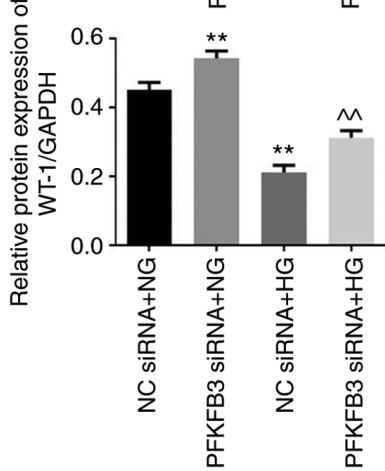

C

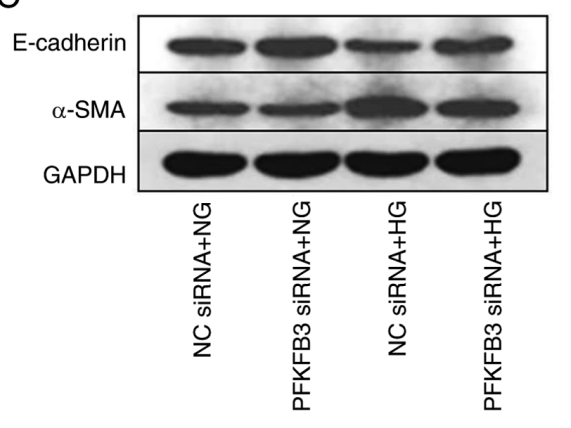

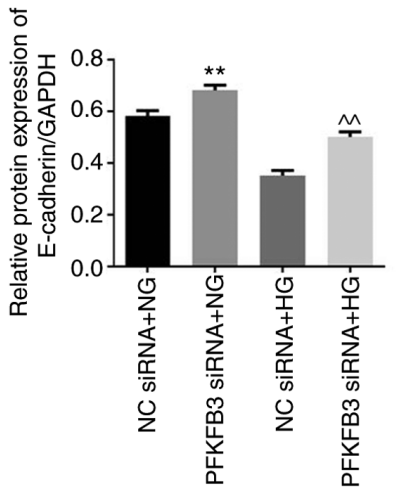

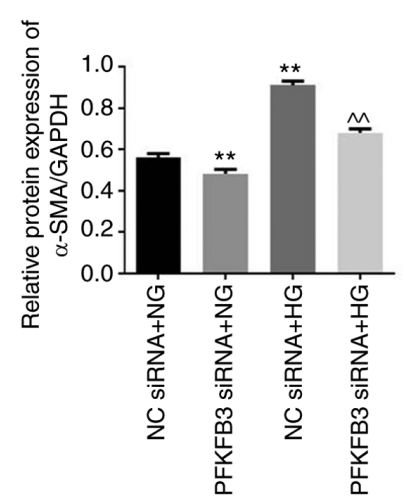

Figure 4. Knockdown of PFKFB3 reverses podocyte injury through inhibition of the epithelial-mesenchymal transition process. Protein expression levels of (A) PFKFB3, (B) WT-1 and (C) E-cadherin and $\alpha$-SMA in MPC5 cells were determined by western blot analysis and normalized to GAPDH. ${ }^{* *} \mathrm{P}<0.01$ vs. $\mathrm{NC}$ siRNA + NG; ${ }^{\wedge} \mathrm{P}<0.01$ vs. NC siRNA + HG. HG, high-glucose; NC, negative control; NG, normal glucose; PFKFB3, 6-phosphofructo-2-kinase/fructose-2,6-biphosphatase 3; SMA, smooth muscle actin; siRNA, small interfering RNA; WT-1, Wilms tumor-1.

Knockdown of PFKFB3 reverses podocyte injury by inhibiting the epithelial-mesenchymal transition (EMT) process. Western blot analysis was performed to measure the expression levels of PFKFB3, WT-1 and EMT-related proteins in the MPC5 cells. Protein expression levels of PFKFB3 in MPC5 cells with NG or HG treatment were significantly decreased by PFKFB3 siRNA compared with NC siRNA + NG or NC siRNA + HG (Fig. 4A). Meanwhile, the expression of PFKFB3 in MPC5 cells was notably upregulated by HG, compared with NG (Fig. 4A). Furthermore, knockdown of PFKFB3 significantly suppressed the inhibitory effect of HG on protein expression level of WT-1 (Fig. 4B). In addition, HG significantly decreased the protein expression levels of E-cadherin and increased those of $\alpha$-SMA (Fig. 4C). However, the effect of HG on these two proteins was significantly reversed following knockdown of PFKFB3. As E-cadherin and $\alpha$-SMA are two key regulators in the EMT process $(30,31)$, these results indicated that knockdown of PFKFB3 may reversed podocyte injury through suppression of the EMT process.
Silencing of PFKFB3 inhibits podocyte injury through activation of autophagy. To determine the association between PFKFB3 and autophagy in HG-treated MPC5 cells, autophagy inhibitors (rapamycin and CQ) were used. Protein expression levels of LC3 II in HG-treated MPC-5 cells were significantly upregulated in cells transfected with PFKFB3 siRNA (Fig. 5A-C). Consistently, the ratio of LC3 II/LC3 I in HG-treated MPC-5 cells was significantly enhanced in the presence of PFKFB3 siRNA compared with in the NC siRNA + HG + rapamycin group. These data suggested that PFKFB3 may exert an inhibitory effect on autophagy. By contrast, knockdown of PFKFB3 significantly decreased the protein expression levels of p62 in MPC5 cells in the presence of $\mathrm{HG}$, whereas CQ or rapamycin (autophagy inhibitors) partially reversed the inhibitory effect on p62 (Fig. 5A and D). In addition, CQ or rapamycin further enhanced the effect of $\mathrm{HG}$ on increased protein expression levels of PFKFB3 in MPC-5 cells (Fig. 5A and E). This showed that downregulation of PFKFB3 induced autophagy in MPC-5 cells. LC3 
A

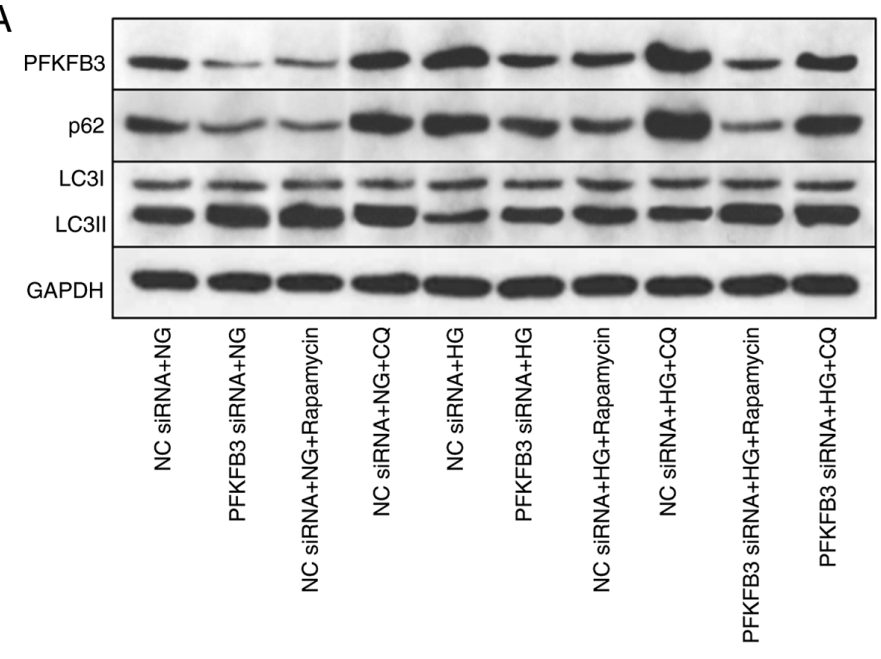

C

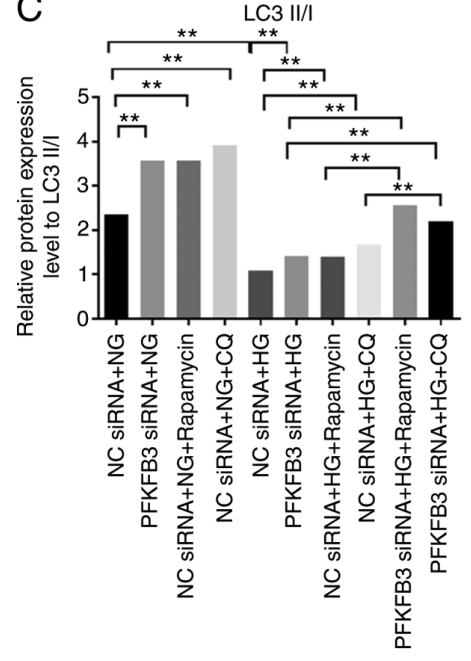

D

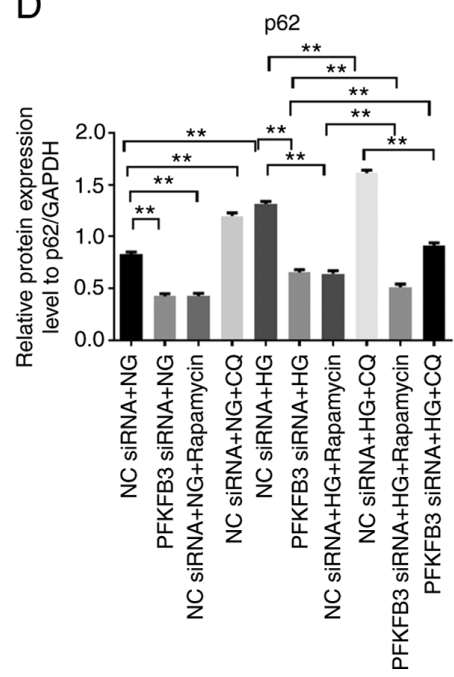

B

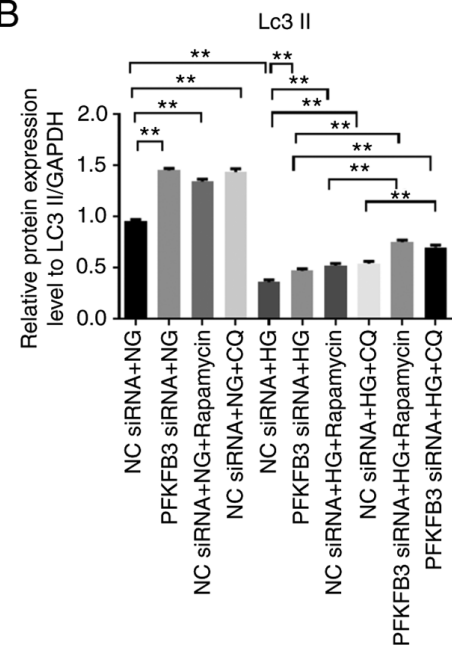

E

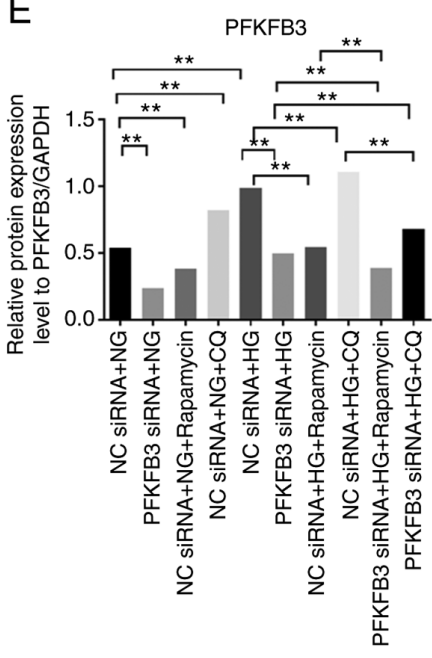

Figure 5. Silencing of PFKFB3 inhibits podocyte injury through activation of autophagy. MPC5 cells were treated with NC siRNA + NG, PFKFB3 siRNA + NG, $\mathrm{NC}$ siRNA + NG + rapamycin, NC siRNA + NG + CQ, NC siRNA + HG, PFKFB3 siRNA + HG, NC siRNA + HG + rapamycin, NC siRNA + HG + CQ, PFKFB3 siRNA + HQ + rapamycin or PFKFB3 siRNA + HQ + CQ. (A) Protein expression levels of PFKFB3, p62, LC3 I and LC3 II were detected by western blot analysis. (B) Relative expression levels of LC3 II were normalized to GAPDH. (C) Ratio of LC3 II/LC3 I was calculated. Relative expression levels of (D) p62 and (E) PFKFB3 were normalized to GAPDH. ${ }^{* *} \mathrm{P}<0.01$. CQ, chloroquine; HG, high-glucose; LC3, light chain 3; NC, negative control; NG, normal glucose; PFKFB3, 6-phosphofructo-2-kinase/fructose-2,6-biphosphatase 3; siRNA, small interfering RNA.

staining indicated that the expression of LC3 (represented by staining intensity) in MPC-5 cells was significantly decreased by HG, which was reversed by PFKFB3 knockdown (Fig. 6). HG treatment significantly inhibited the levels of p-AMPK $\alpha$ and SIRT1, and significantly upregulated the expression of p-mTOR; these effects were reversed following PFKFB3 knockdown (Fig. 7A-D). Rapamycin significantly enhanced the effect of PFKFB3 siRNA on p-mTOR expression, whereas HG-mediated p-AMPK $\alpha$ and SIRT1 expression was not significantly affected by CQ or rapamycin (Fig. 7A-D). Taken together, these results indicated that silencing of PFKFB3 may inhibit podocyte injury by activating autophagy.

Effect of PFKFB3 siRNA on apoptosis and migration of $H G$-treated podocytes was reversed by autophagy inhibitor. To verify the association between PFKFB3 and cell autophagy in MPC-5 cells, CCK8, flow cytometry and Transwell assays were performed. Inhibition of PFKFB3 significantly increased primary podocyte viability (Fig. S3A) and suppressed podocyte apoptosis (Fig. S3B) and MPC-5 cell migration (Fig. 8A) in the presence of NG, compared with NG + NC siRNA. In addition, rapamycin inhibited the migration of MPC5 cells compared with in the NG/HG + NC siRNA groups (Fig. 8A). HG increased cell migration compared with the NG + NC siRNA group (Fig. 8A). Besides, HG-induced increase of cell migration was reversed by PFKFB3 siRNA or rapamycin, whereas it was enhanced by CQ (Fig. 8A). Meanwhile, the inhibitory effect of PFKFB3 siRNA on migration of HG-treated MPC5 cells was enhanced by rapamycin but slightly rescued by CQ (Fig. 8A). Furthermore, the HG-induced decrease of cell area was notably reversed by knockdown of PFKFB3. However, CQ notably reversed the inhibitory effect of PFKFB3 siRNA on cell morphology (Fig. 8B). In summary, the effect of PFKFB3 siRNA on apoptosis and migration of HG-treated podocytes was reversed by an autophagy inhibitor.

Autophagy inhibitor reverses the effect of PFKFB3 siRNA on the viability and DAG of $H G$-treated podocytes. Glucose 

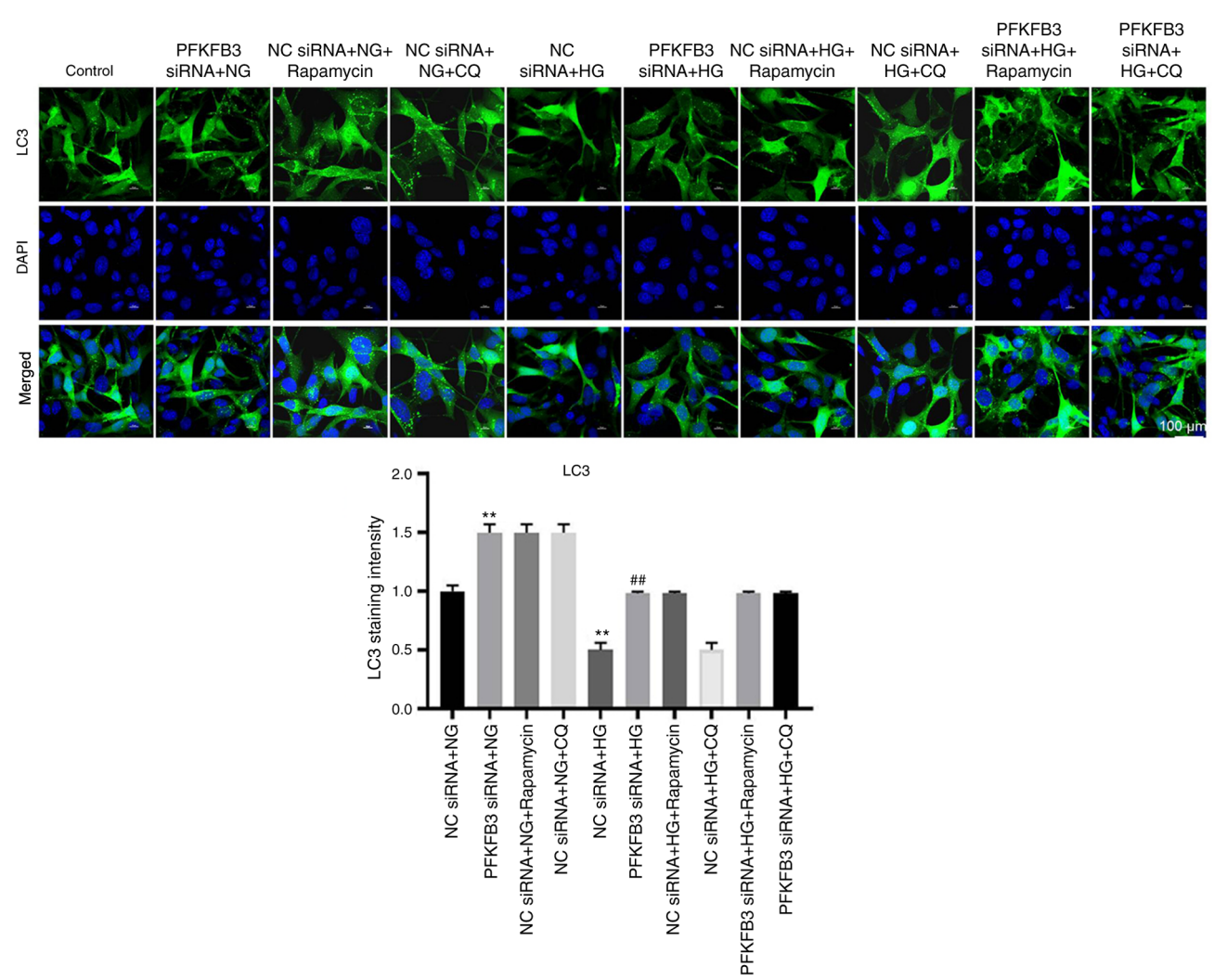

Figure 6. Silencing of PFKFB3 promotes expression of LC3 in HG-treated MPC5 cells. The expression levels of LC3 in MPC5 cells were detected by LC3 staining. ${ }^{* *} \mathrm{P}<0.01$ vs. NC siRNA + NG; ${ }^{\# \#} \mathrm{P}<0.01$ vs. NC siRNA + HG. CQ, chloroquine; HG, high-glucose; LC3, light chain 3; NC, negative control; siRNA, small interfering RNA; NG, normal glucose; PFKFB3, 6-phosphofructo-2-kinase/fructose-2,6-biphosphatase 3.
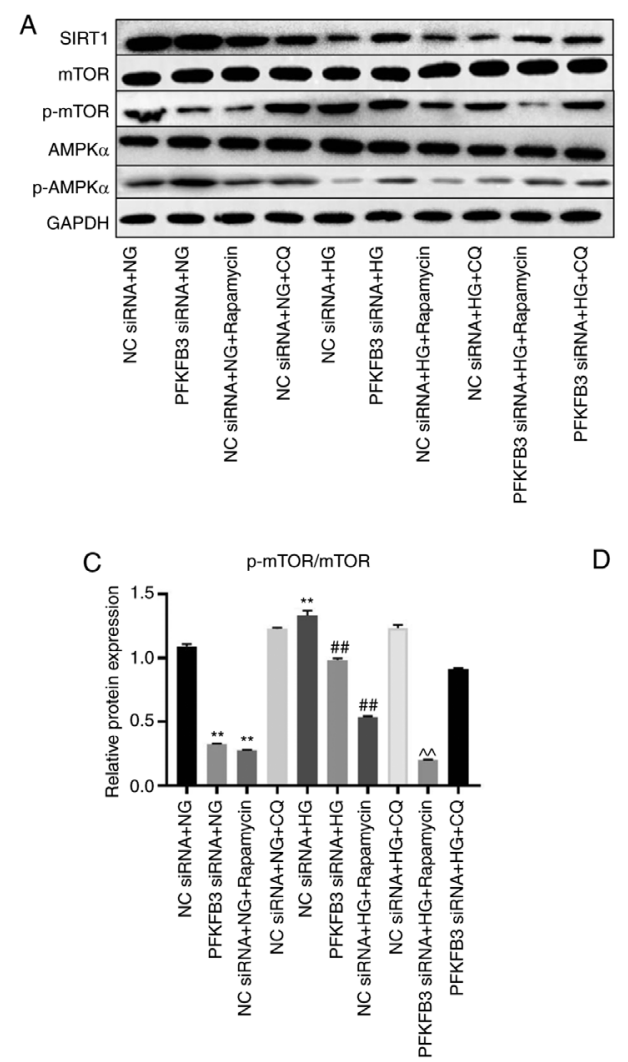

\section{B}

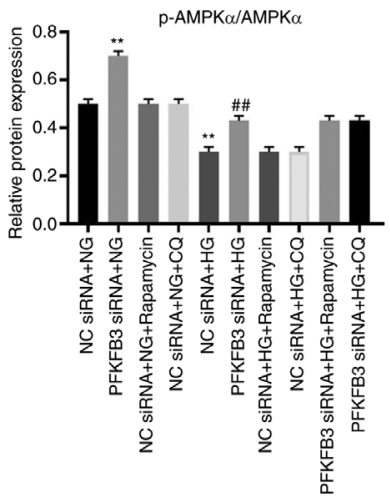

D

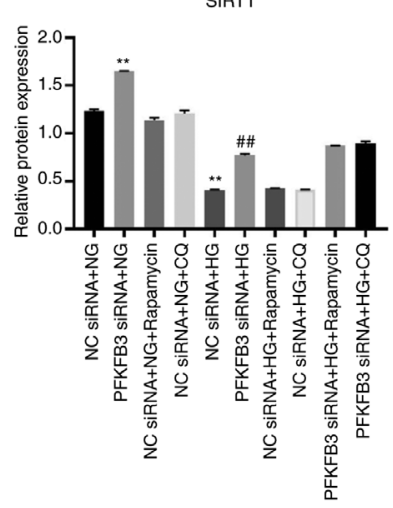

Figure 7. Silencing of PFKFB3 induces autophagy by mediating p-mTOR and p-AMPK $\alpha$. (A) Protein expression levels of SIRT1, mTOR, p-mTOR, p-AMPKa and AMPK $\alpha$ in MPC5 cells were investigated by western blotting. (B) Relative expression of p-AMPK $\alpha$ was normalized to AMPK $\alpha$. (C) Relative expres-

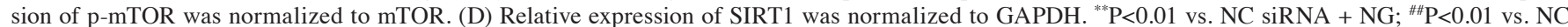
siRNA + HG; ${ }^{\wedge} \mathrm{P}<0.01$ vs. PFKFB3 siRNA + HG. CQ, chloroquine; HG, high-glucose; NG, normal glucose; NC, negative control; p-, phosphorylated; PFKFB3, 6-phosphofructo-2-kinase/fructose-2,6-biphosphatase 3; siRNA, small interfering RNA; SIRT1, sirtuin 1. 
A
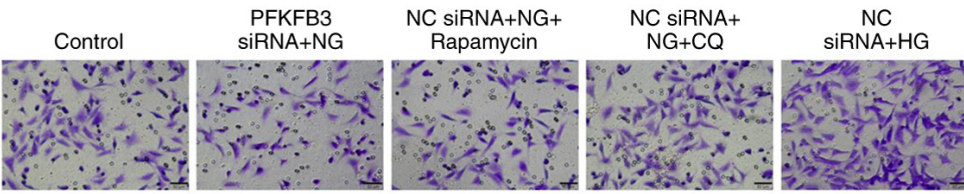

PFKFB3 SiRNA+HG

$\mathrm{NC}$ siRNA+HG+

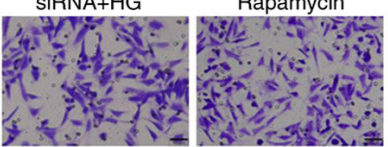

$\mathrm{HG}+\mathrm{CQ}$ Rapamycin $\mathrm{HG}+\mathrm{CQ}$
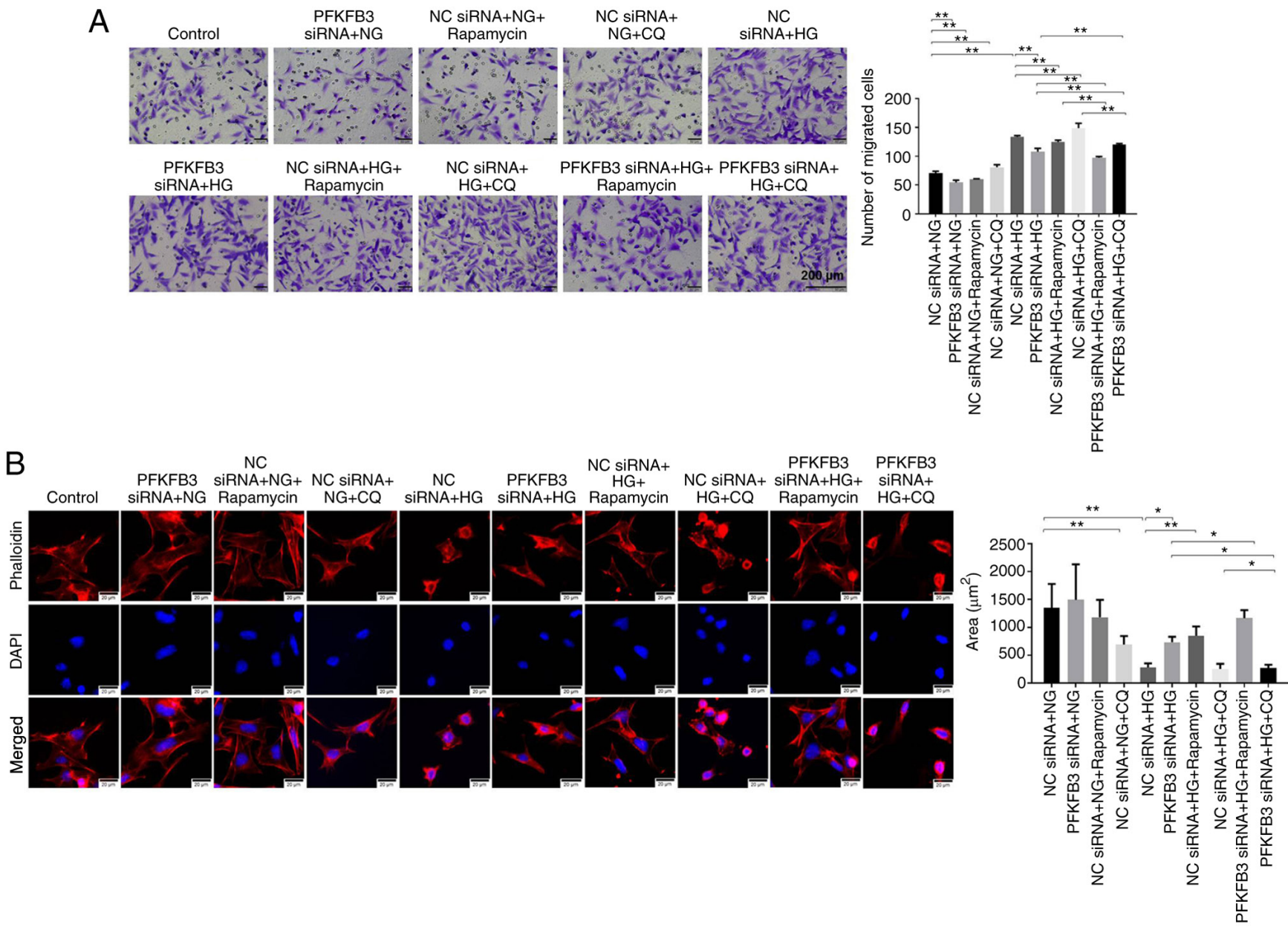

Figure 8. HG-induced increase in cell migration is reversed by PFKFB3 siRNA. (A) Cell migration was tested by Transwell assay. Scale bar, $200 \mu \mathrm{m}$. (B) Phalloidin immunofluorescence staining was performed to detect the cytoskeleton and the area of MPC5 cells was calculated. Scale bar, $20 \mu \mathrm{m}$. ${ }^{*} \mathrm{P}<0.05$, ${ }^{* *} \mathrm{P}<0.01$. CQ, chloroquine; HG, high-glucose; NC, negative control; NG, normal glucose; PFKFB3, 6-phosphofructo-2-kinase/fructose-2,6-biphosphatase 3; siRNA, small interfering RNA.

uptake of MPC-5 cells was detected. The inhibitory effect of PFKFB3 siRNA on glucose uptake of HG-MPC-5 cells was partially reversed by CQ after 24, 48 and $72 \mathrm{~h}$ of incubation (Fig. 9A). Similarly, knockdown of PFKFB3 significantly reversed the $\mathrm{HG}$-induced increase in DAG levels in MPC5 cells; this was partially rescued by CQ (Fig. 9B). CCK-8 assay results demonstrated that downregulation of PFKFB3 significantly increased the viability of MPC5 cells in the presence of HG compared with the NC siRNA + HG group, and the effect was notably inhibited by CQ (Fig. 9C). Furthermore, western blot analysis was used to detect the expression levels of synaptopodin and WT-1 in the MPC5 cells. Protein expression levels of synaptopodin and WT-1 in the presence of HG were increased following inhibition of PFKFB3, although this was only significant for synaptopodin expression levels (Fig. 9D). However, the effect of PFKFB3 siRNA (in the PFKFB3 siRNA + HG group) on WT-1 levels was slightly rescued by CQ (in the PFKFB3 siRNA + HG + CQ group).

\section{Discussion}

A number of previous studies have demonstrated that mRNA expression levels are dysregulated in a variety of serious human diseases, including DN (32-34). In the present study, the molecular mechanisms by which PFKFB3 siRNA alleviated HG-induced podocyte injury were investigated. PFKFB3 expression levels were upregulated in MPC5 cells following HG treatment. In addition, it has been reported that induction of autophagy alleviates podocyte injury (35), which is consistent with the results of the present study. This may be because activation of autophagy may inhibit cell apoptosis (22).

A recent study demonstrated that multiple distinct mRNAs (SIRT1, DNMT1, etc.) modulate the progression of DN by targeting a wide range of signaling pathways associated with HG $(36,37)$. A range of interrelated factors, including genetics, ischemia, inflammation and hypoxia, have been shown to promote DN progression (38-40). Numerous mRNAs (SIRT3, LC3, etc.) modulate DAG and glucose uptake in cells by targeting the AMPK signaling pathway $(41,42)$. The present study explored the detailed mechanism by which PFKFB3 regulates the progression of DN.

In the present study, the effect of PFKFB3 on autophagy was investigated. Autophagy is the biological cellular process by which intracellular components undergo lysosome-mediated self-digestion and recycling (43). Autophagy is associated with the progression of numerous diseases, such as inflammation and cancer (44-46). Previous reports have indicated 

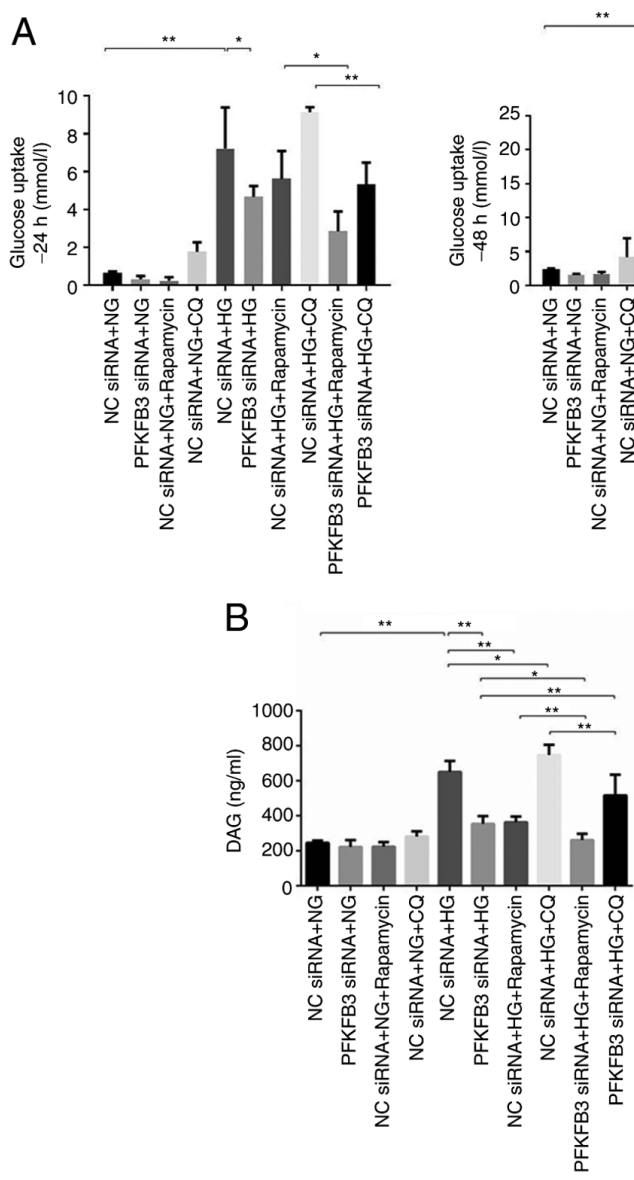
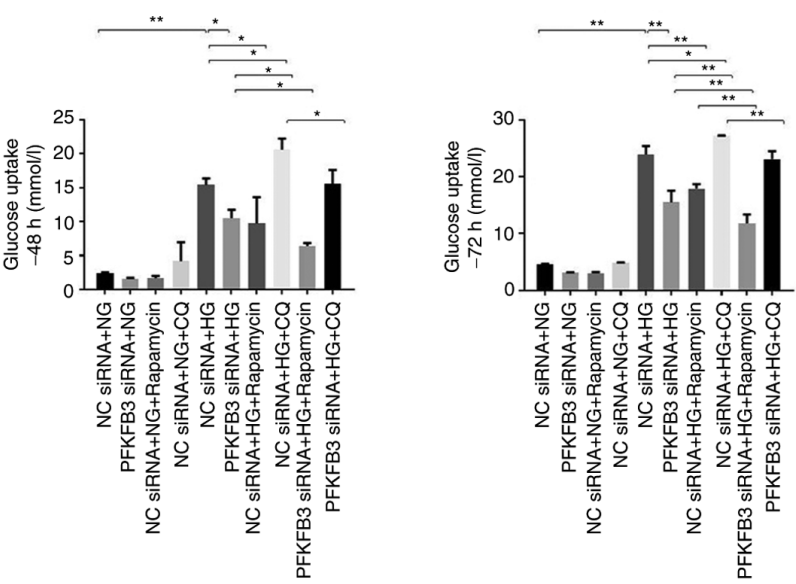

C

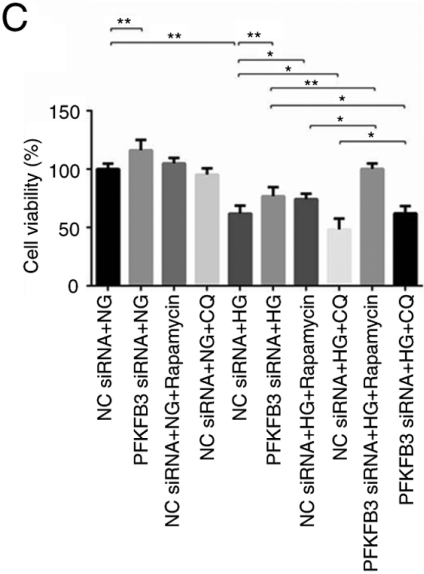

D

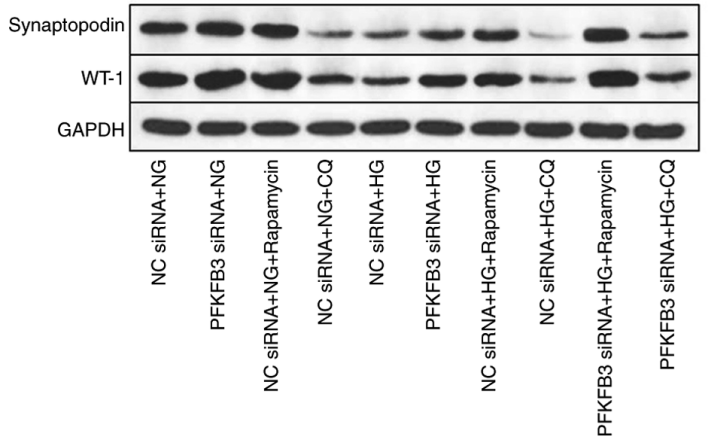

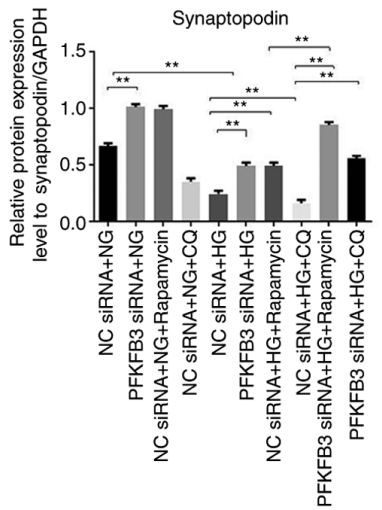

WT-1

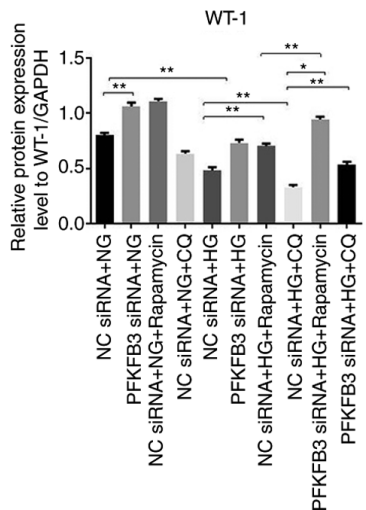

Figure 9. CQ reverses the effect of PFKFB3 siRNA on podocyte proliferation. (A) Glucose uptake in MPC5 cells was calculated at 24, 48 or 72 h. (B) Levels of DAG in MPC5 cells were tested using a microplate reader. (C) Cell viability was determined by Cell Counting Kit-8 assay. (D) Protein expression levels of synaptopodin and WT-1 in MPC5 cells were examined by western blot analysis and normalized to GAPDH. "P<0.05, ${ }^{\text {** }} \mathrm{P}<0.01$. CQ, chloroquine; DAG, diacylglycerol; HG, high-glucose; NC, negative control; NG, normal glucose; PFKFB3, 6-phosphofructo-2-kinase/fructose-2,6-biphosphatase 3; siRNA, small interfering RNA; WT-1, Wilms tumor-1.

that activation of autophagy ameliorates the symptoms of DN (47-49), which was similar to the results of the present in vitro study; knockdown of PFKFB3 activated autophagy. Results from the present study also indicated that PFKFB3 siRNA may mediate the expression of LC3, p-mTOR, p-AMPK $\alpha$ and SIRT1 in podocytes. As these proteins are crucial factors during cell autophagy $(50,51)$, it may be concluded that PFKFB3 knockdown induced cell autophagy through the modulation of these protein expressions. By contrast, La Belle Flynn et al (52) revealed that inhibition of PFKFB3 significantly inhibits autophagy in the progression of breast cancer. This discrepancy may be due to different cell types being investigated.

During the activation of autophagy flux, LC3 II accumulation increases, while soluble p62 is decreased $(53,54)$. In addition, certain reports have indicated that p62 downregulation does not always activate autophagic flux as LC3 I may not be converted to LC3 II $(55,56)$. In the present study, LC3 II accumulation was increased in the PFKFB3 siRNA + HG + CQ group compared with the NC siRNA + HG + CQ group. Moreover, p62 accumulation was notably inhibited in the PFKFB3 siRNA + HG + CQ group 
compared with the NC siRNA + HG + CQ group. Thus, these data suggested that PFKFB3 siRNA activated autophagic flux in MPC5 cells. Moreover, the present results suggested that PFKFB3 silencing reversed HG-induced podocyte apoptosis. It has been confirmed that autophagy serves an important role in cell apoptosis (57). Despite their distinct mechanisms and functions, apoptosis and autophagy are closely associated. Autophagy usually mediates the cell apoptosis (58). Thus, it can be concluded that PFKFB3 knockdown reversed HG-induced podocyte apoptosis by inducing autophagy.

It was observed in the present study that CQ and rapamycin did not always have the same effect on PFKFB3 siRNA-mediated DN. Rapamycin is known to function as an inhibitor of the mTOR pathway, whereas CQ can suppress autophagy via inhibiting the binding between autophagosomes and lysosomes $(59,60)$. Thus, the different mechanism by which CQ and rapamycin regulate autophagy may result in different results. Notably, CQ and rapamycin were shown to have no effect on LC3 levels. LC3 is involved in the formation of autophagosomes, and it is not associated with the mTOR pathway or the binding between autophagosomes and lysosomes $(55,56)$; therefore, this may explain why CQ and rapamycin had no effect on LC3 levels.

There are certain limitations in the present study. First, in vivo experiments are required to further support the results. In addition, the association between TGF- $\beta$ and the EMT process in podocytes is unclear. Therefore, more investigations are required in the future.

In conclusion, knockdown of PFKFB3 may protect podocytes from HG injury by inducing autophagy. Thus, PFKFB3 may serve as a novel target for the treatment of $\mathrm{DN}$.

\section{Acknowledgements}

Not applicable.

\section{Funding}

No funding was received.

\section{Availability of data and materials}

The datasets used and/or analyzed during the current study are available from the corresponding author on reasonable request.

\section{Authors' contributions}

ZZ and QL conceived and supervised the study. QL and JS designed the study. ZB and WW performed the experiments and analyzed the data. QL and ZZ confirm the authenticity of all the raw data. All authors read and approved the final version of the manuscript.

\section{Ethics approval and consent to participate}

Not applicable.

\section{Patient consent for publication}

Not applicable.

\section{Competing interests}

These authors declare they have no competing interests.

\section{References}

1. Cankurtaran V, Inanc M, Tekin K and Turgut F: Retinal microcirculation in predicting diabetic nephropathy in type 2 diabetic patients without retinopathy. Ophthalmologica 243: 271-279, 2020.

2. Elbassuoni EA, Aziz NM and Habeeb WN: The role of activation of $\mathrm{K}^{\text {ATP }}$ channels on hydrogen sulfide induced renoprotective effect on diabetic nephropathy. J Cell Physiol 235: 5223-5228, 2020.

3. Shao J, Xu H, Wu X and Xu Y: Epigenetic activation of CTGF transcription by high glucose in renal tubular epithelial cells is mediated by myocardin-related transcription factor A. Cell Tissue Res 379: 549-559, 2020.

4. Al Shawaf E, Abu-Farha M, Devarajan S, Alsairafi Z, Al-Khairi I, Cherian P, Ali H, Mathur A, Al-Mulla F, Al Attar A, et al: ANGPTL4: A predictive marker for diabetic nephropathy. J Diabetes Res 2019: 4943191, 2019.

5. Du L, Wang J, Chen Y, Li X, Wang L, Li Y, Jin X, Gu X, Hao M, Zhu X, et al: Novel biphenyl diester derivative AB-38b inhibits NLRP3 inflammasome through Nrf2 activation in diabetic nephropathy. Cell Biol Toxicol 36: 243-260, 2020.

6. Liu L, Chen H, Yun J, Song L, Ma X, Luo S and Song Y: miRNA-483-5p targets HDCA4 to regulate renal tubular damage in diabetic nephropathy. Horm Metab Res 2021 (Epub ahead of print). doi: $10.1055 / \mathrm{a}-1480-7519$.

7. Clem BF, O'Neal J, Tapolsky G, Clem AL, Imbert-Fernandez Y, Kerr DA II, Klarer AC, Redman R, Miller DM, Trent JO, et al: Targeting 6-phosphofructo-2-kinase (PFKFB3) as a therapeutic strategy against cancer. Mol Cancer Ther 12: 1461-1470, 2013.

8. Lu L, Chen Y and Zhu Y: The molecular basis of targeting PFKFB3 as a therapeutic strategy against cancer. Oncotarget 8: 62793-62802, 2017.

9. Sarkar Bhattacharya S, Thirusangu P, Jin L, Roy D, Jung D, Xiao Y, Staub J, Roy B, Molina JR and Shridhar V: PFKFB3 inhibition reprograms malignant pleural mesothelioma to nutrient stress-induced macropinocytosis and ER stress as independent binary adaptive responses. Cell Death Dis 10: 725, 2019.

10. Tao L, Yu H, Liang R, Jia R, Wang J, Jiang K and Wang Z: Rev-erb $\alpha$ inhibits proliferation by reducing glycolytic flux and pentose phosphate pathway in human gastric cancer cells. Oncogenesis 8: 57, 2019.

11. Wade SM, Ohnesorge N, McLoughlin H, Biniecka M, Carter SP, Trenkman M, Cunningham CC, McGarry T, Canavan M, Kennedy BN, et al: Dysregulated miR-125a promotes angiogenesis through enhanced glycolysis. EBioMedicine 47: 402-413, 2019.

12. Li YJ, Lei YH, Yao N, Wang CR, Hu N, Ye WC, Zhang DM and Chen ZS: Autophagy and multidrug resistance in cancer. Chin J Cancer 36: 52, 2017.

13. Ravanan P, Srikumar IF and Talwar P: Autophagy: The spotlight for cellular stress responses. Life Sci 188: 53-67, 2017.

14. Zhang L: Pharmacokinetics and drug delivery systems for puerarin, a bioactive flavone from traditional Chinese medicine. Drug Deliv 26: 860-869, 2019.

15. Lin X, Chen Y, Zhang P, Chen G, Zhou Y and Yu X: The potential mechanism of postoperative cognitive dysfunction in older people. Exp Gerontol 130: 110791, 2020.

16. Son YO: Molecular mechanisms of nickel-induced carcinogenesis. Endocr Metab Immune Disord Drug Targets 20: 1015-1023, 2020.

17. Tang Q, Chen Z, Zhao L and Xu H: Circular RNA hsa circ_0000515 acts as a miR-326 sponge to promote cervical cancer progression through up-regulation of ELK1. Aging (Albany NY) 11: 9982-9999, 2019.

18. Wang Q, Li R, Xiao Z and Hou C: Lycopene attenuates high glucose-mediated apoptosis in MPC5 podocytes by promoting autophagy via the PI3K/AKT signaling pathway. Exp Ther Med 20: 2870-2878, 2020.

19. Sawada N and Arany Z: Metabolic regulation of angiogenesis in diabetes and aging. Physiology (Bethesda) 32: 290-307, 2017.

20. Mizukami H, Osonoi S, Takaku S, Yamagishi SI, Ogasawara S, Sango K, Chung S and Yagihashi S: Role of glucosamine in development of diabetic neuropathy independent of the aldose reductase pathway. Brain Commun 2: fcaa168, 2020. 
21. Liu B, He X, Li S, Xu B, Birnbaumer L and Liao Y: Deletion of diacylglycerol-responsive TRPC genes attenuates diabetic nephropathy by inhibiting activation of the TGF $\beta 1$ signaling pathway. Am J Transl Res 9: 5619-5630, 2017.

22. Chen JN, Li T, Cheng L, Qin TS, Sun YX, Chen CT, He YZ, Liu G, Yao D, Wei Y, et al: Synthesis and in vitro anti-bladder cancer activity evaluation of quinazolinyl-arylurea derivatives Eur J Med Chem 205: 112661, 2020.

23. Shen Y, Tong ZW, Zhou Y, Sun Y, Xie Y, Li R and Liu H: Inhibition of lncRNA-PAX8-AS1-N directly associated with VEGF/TGF- $31 / 8-O h d G$ enhances podocyte apoptosis in diabetic nephropathy. Eur Rev Med Pharmacol Sci 24: 6864-6872, 2020.

24. He J, Gao HX, Yang N, Zhu XD, Sun RB, Xie Y, Zeng CH, Zhang JW, Wang JK, Ding F, et al: The aldose reductase inhibitor epalrestat exerts nephritic protection on diabetic nephropathy in $\mathrm{db} / \mathrm{db}$ mice through metabolic modulation. Acta Pharmacol Sin 40: 86-97, 2019 .

25. Albers JW and Pop-Busui R: Diabetic neuropathy: Mechanisms, emerging treatments, and subtypes. Curr Neurol Neurosci Rep 14: 473, 2014

26. Qian X, Xu W, Xu J, Shi Q, Li J, Weng Y, Jiang Z, Feng L, Wang X, Zhou J and Jin H: Enolase 1 stimulates glycolysis to promote chemoresistance in gastric cancer. Oncotarget 8 : 47691-47708, 2017.

27. Almacellas E, Pelletier J, Manzano A, Gentilella A, Ambrosio S, Mauvezin C and Tauler A: Phosphofructokinases axis controls glucose-dependent mTORC1 activation driven by E2F1. iScience 20: 434-448, 2019.

28. ElGamal $\mathrm{H}$ and Munusamy S: Aldose reductase as a drug target for treatment of diabetic nephropathy: Promises and challenges. Protein Pept Lett 24: 71-77, 2017.

29. Liu YW, Cheng YQ, Liu XL, Hao YC, Li Y, Zhu X, Zhang F and Yin XX: Mangiferin upregulates glyoxalase 1 through activation of Nrf2/ARE signaling in central neurons cultured with high glucose. Mol Neurobiol 54: 4060-4070, 2017.

30. Wong SHM, Fang CM, Chuah LH, Leong CO and Ngai SC: E-cadherin: Its dysregulation in carcinogenesis and clinical implications. Crit Rev Oncol Hematol 121: 11-22, 2018.

31. Saitoh M: Involvement of partial EMT in cancer progression. J Biochem 164: 257-264, 2018

32. Gao C, Chen J, Fan F, Long Y, Tang S, Jiang C, Wang J, Xu Y and Xu Y: RIPK2-mediated autophagy and negatively regulated ROS-NLRP3 inflammasome signaling in GMCs stimulated with high glucose. Mediators Inflamm 2019: 6207563, 2019.

33. Gong J, Zhan H, Li Y, Zhang W, Jin J and He Q: Kruppel-like factor 4 ameliorates diabetic kidney disease by activating autophagy via the mTOR pathway. Mol Med Rep 20: 3240-3248, 2019.

34. Sankrityayan H, Oza MJ, Kulkarni YA, Mulay SR and Gaikwad AB: ER stress response mediates diabetic microvascular complications. Drug Discov Today 24: 2247-2257, 2019.

35. Tu Q, Li Y, Jin J, Jiang X, Ren Y and He Q: Curcumin alleviates diabetic nephropathy via inhibiting podocyte mesenchyma transdifferentiation and inducing autophagy in rats and MPC5 cells. Pharm Biol 57: 778-786, 2019.

36. Hou Y, Lin S, Qiu J, Sun W, Dong M, Xiang Y, Wang L and Du P NLRP3 inflammasome negatively regulates podocyte autophagy in diabetic nephropathy. Biochem Biophys Res Commun 521 791-798, 2020

37. Syed AA, Reza MI, Garg R, Goand UK and Gayen JR: Cissus quadrangularis extract attenuates diabetic nephropathy by altering SIRT1/DNMT1 axis. J Pharm Pharmacol: Jun 15, 2021 (Epub ahead of print). doi: 10.1093/jpp/rgab078.

38. Guo L, Tan K, Luo Q and Bai X: Dihydromyricetin promotes autophagy and attenuates renal interstitial fibrosis by regulating miR-155-5p/PTEN signaling in diabetic nephropathy. Bosn J Basic Med Sci 20: 372-380, 2020.

39. Bu J, Shi S, Wang HQ, Niu XS, Zhao ZF, Wu WD, Zhang XL, Ma Z, Zhang YJ, Zhang $\mathrm{H}$ and Zhu Y: Acacetin protects against cerebral ischemia-reperfusion injury via the NLRP3 signaling pathway. Neural Regen Res 14: 605-612, 2019

40. Zhuang L, Jin G, Hu X, Yang Q and Shi Z: The inhibition of SGK1 suppresses epithelial-mesenchymal transition and promotes renal tubular epithelial cell autophagy in diabetic nephropathy. Am J Transl Res 11: 4946-4956, 2019.

41. Wang Y, Zhang X, Wang P, Shen Y, Yuan K, Li M, Liang W and Que H: Sirt3 overexpression alleviates hyperglycemia-induced vascular inflammation through regulating redox balance, cell survival, and AMPK-mediated mitochondrial homeostasis. J Recept Signal Transduct Res 39: 341-349, 2019.
42. Woo CY, Kc R, Kim M, Kim HS, Baek JY and Koh EH: Autophagic flux defect in diabetic kidney disease results in megamitochondria formation in podocytes. Biochem Biophys Res Commun 521: 660-667, 2020.

43. Yang $\mathrm{Z}$ and Klionsky DJ: Eaten alive: A history of macroautophagy. Nat Cell Biol 12: 814-822, 2010.

44. Dasgupta S: Mitochondrion: I am more than a fuel server. Ann Transl Med 7: 594, 2019.

45. Wu Q, Tian AL, Li B, Leduc M, Forveille S, Hamley P, Galloway W, Xie W, Liu P, Zhao L, et al: IGF1 receptor inhibition amplifies the effects of cancer drugs by autophagy and immune-dependent mechanisms. J Immunother Cancer 9: e002722, 2021

46. Kommalapati VK, Kumar D and Tangutur AD: Quisinostat mediated autophagy is associated with differentiation in neuroblastoma SK-N-SH cells. Mol Biol Rep 48: 4973-4979, 2021.

47. Xu J, Liu LQ, Xu LL, Xing Y and Ye S: Metformin alleviates renal injury in diabetic rats by inducing Sirt1/FoxO1 autophagic signal axis. Clin Exp Pharmacol Physiol 47: 599-608, 2020.

48. Ye X, Zhou XJ and Zhang H: Autophagy in immune-related renal disease. J Immunol Res 2019: 5071687, 2019.

49. Alvarez-Cilleros D, Lopez-Oliva ME, Martin MA and Ramos S: Cocoa ameliorates renal injury in Zucker diabetic fatty rats by preventing oxidative stress, apoptosis and inactivation of autophagy. Food Funct 10: 7926-7939, 2019.

50. Chen L, Zhao L, Samanta A, Mahmoudi SM, Buehler T, Cantilena A, Vincent RJ, Girgis M, Breeden J, Asante S, et al: STAT3 balances myocyte hypertrophy vis-a-vis autophagy in response to Angiotensin II by modulating the AMPK $\alpha / \mathrm{mTOR}$ axis. PLoS One 12: e0179835, 2017.

51. Zhang P, Liu X, Li H, Chen Z, Yao X, Jin J and Ma X: TRPC5-induced autophagy promotes drug resistance in breast carcinoma via CaMKK $\beta / \mathrm{AMPK} \alpha / \mathrm{mTOR}$ pathway. Sci Rep 7: 3158, 2017.

52. La Belle Flynn A, Calhoun BC, Sharma A, Chang JC, Almasan A and Schiemann WP: Autophagy inhibition elicits emergence from metastatic dormancy by inducing and stabilizing Pfkfb3 expression. Nat Commun 10: 3668, 2019.

53. Chu CW, Ko HJ, Chou CH, Cheng TS, Cheng HW, Liang YH, Lai YL, Lin CY, Wang C, Loh JK, et al: Thioridazine enhances P62-Mediated autophagy and apoptosis through Wnt/ $\beta$-catenin signaling pathway in glioma cells. Int J Mol Sci 20: 473, 2019.

54. Guo FX, Wu Q, Li P, Zheng L, Ye S, Dai XY, Kang CM, Lu JB, $\mathrm{Xu} \mathrm{BM}, \mathrm{Xu}$ YJ, et al: The role of the LncRNA-FA2H-2-MLKL pathway in atherosclerosis by regulation of autophagy flux and inflammation through mTOR-dependent signaling. Cell Death Differ 26: 1670-1687, 2019.

55. Guo H, Ding H, Yan Y, Chen Q, Zhang J, Chen B and Cao J: Intermittent hypoxia-induced autophagy via AMPK/mTOR signaling pathway attenuates endothelial apoptosis and dysfunction in vitro. Sleep Breath 2021 (Epub ahead of print).

56. Chen J, Wang L, Liu WH, Shi J, Zhong Y, Liu SJ and Liu SM: Aspirin protects human coronary artery endothelial cells by inducing autophagy. Physiol Int 107: 294-305, 2020.

57. Wang Z, Liu N, Liu K, Zhou G, Gan J, Wang Z, Shi T, He W, Wang L, Guo T, et al: Autophagy mediated CoCrMo particle-induced peri-implant osteolysis by promoting osteoblast apoptosis. Autophagy 11: 2358-2369, 2015.

58. Hale AN, Ledbetter DJ, Gawriluk TR and Rucker EB III: Autophagy: Regulation and role in development. Autophagy 9: 951-972, 2013

59. Corral-Ramos C, Barrios R, Ayté J and Hidalgo E: TOR and MAP kinase pathways synergistically regulate autophagy in response to nutrient depletion in fission yeast. Autophagy: Jun 23, 2021 (Epub ahead of print). doi: 10.1080/15548627.2021.1935522.

60. Gray JP, Uddin MN, Chaudhari R, Sutton MN, Yang H, Rask P, Locke H, Engel BJ, Batistatou N, Wang J, et al: Directed evolution of cyclic peptides for inhibition of autophagy. Chem Sci 12: 3526-3543, 2021

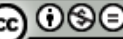

This work is licensed under a Creative Commons Attribution-NonCommercial-NoDerivatives 4.0 International (CC BY-NC-ND 4.0) License. 